\title{
A standardized extract from Paeonia lactiflora and Astragalus membranaceus induces apoptosis and inhibits the proliferation, migration and invasion of human hepatoma cell lines
}

\author{
JING-JING WU, WU-YI SUN, SHAN-SHAN HU, SEN ZHANG and WEI WEI
}

\begin{abstract}
Institute of Clinical Pharmacology, Anhui Medical University, Key Laboratory of Antiinflammatory and Immune Medicine, Ministry of Education, Engineering Technology Research Center of Anti-inflammatory and Immunodrugs in Anhui Province, Hefei, Anhui 230032, P.R. China
\end{abstract}

Received June 30, 2013; Accepted August 9, 2013

DOI: 10.3892/ijo.2013.2085

\begin{abstract}
Paeonia lactiflora and Astragalus membranaceus are two traditional Chinese medicines, which are commonly used in Chinese herb prescription to treat liver diseases. The protective effects of the extract prepared from the roots of Paeonia lactiflora and Astragalus membranaceus (PAE) on liver fibrosis have been demonstrated in previous studies. However, its effect on hepatocellular carcinoma (HCC) has not been investigated to date. In this study, the effects of PAE on the apoptosis, proliferation, migration and invasion of the human hepatoma cell lines HepG2 and SMMC-7721 were investigated. Our data demonstrated that treatment with PAE (50-200 mg/l) caused an inhibitory effect on the proliferation of the hepatoma cell lines HepG2 and SMMC-7721. Furthermore, PAE induced apoptosis of HepG2 cells and SMMC-7721 cells, which was demonstrated by PI staining. In addition, immunocytochemistry and western blotting showed that PAE significantly decreased the expression of Bcl-2, while the expression of Bax and cleaved caspase-3 in HepG2 cells and SMMC-7721 cells was significantly increased after treatment with PAE. These results clearly demonstrated that PAE induced hepatoma cell apoptosis through increasing the Bax-to-Bcl-2 ratio and upregulating the activation of caspase-3. In addition, the results of wound healing assay and Matrigel invasion assay showed that PAE displayed inhibitory activity on the migration and invasion of HCC cells. Taken together, the present data provides evidence that PAE is a potent antineoplastic drug candidate for the treatment of HCC.
\end{abstract}

Correspondence to: Professor Wu-Yi Sun or Professor Wei Wei, Institute of Clinical Pharmacology, Anhui Medical University, Meishan Road, Hefei, Anhui 230032, P.R. China

E-mail: sunwuyi51@yahoo.com.cn

E-mail:wwei@ahmu.edu.cn

Key words: Paeonia lactiflora, Astragalus membranaceus, hepatocellular carcinoma, apoptosis, HepG2, SMMC-7721

\section{Introduction}

Hepatocellular carcinoma (HCC) is the fifth most common cancer and the third most common cause of cancer-related mortality worldwide, which is often associated with longstanding chronic liver inflammation and cirrhosis (1-3). Conventional chemotherapy is one of the common strategies in HCC treatment (4). However, chemotherapeutic drugs are often accompanied by multiple side effects. In the tradition of eastern medicine, natural drugs have made a significant contribution to the treatment of liver diseases. Chinese herbal medicines are serving as a rich source for discovering new therapeutic agents in tumor because of their effectiveness and low side effects $(5,6)$. Therefore, the search for new effective agents possibly from natural herbs with the ability of directly inhibit HCC cell proliferation or to induce HCC cells apoptosis is urgent.

Paeonia lactiflora pall root, a traditional Chinese herb, was recognized as a component of effective prescriptions for treatment of liver disease (7). Total glucosides of peony (TGP) which consists of paeoniflorin, albiflorin, benzoylpaeoniflorin, oxypaeoniflorin, paeonin, etc., extracted from Paeonia lactiflora pall root, has been recognized as the effective herbs which have been used in the treatment of hepatitis and other chronic liver disease (8). The anti-inflammatory, antioxidative, anti-hepatic injury and immunoregulatory activities of TGP have been extensively proved by our laboratory for several years $(8,9)$. Our previous studies have demonstrated that TGP could significantly inhibit proliferation of human hepatoma cells $(10,11)$. In addition, the main bioactive components of TGP, paeoniflorin, has been reported to exert antitumor activity against a number of malignancies including non-small cell lung cancer, gastric cancer, leukemia, and cervical cancer (12-14). Astragalus membranaceus, another popular traditional Chinese herb, exhibited significant growthinhibitory, proapoptotic and angiogenesis-suppression effects in human cancer cells in pharmacological experiments and relevant clinical reports $(15,16)$. In vitro studies of our laboratory demonstrated that Radix Astragali, its extracts, as well 
as its active ingredients such as astragalosides, significantly induced apoptosis and inhibited invasion of tumor cells $(17,18)$. In traditional oriental medicine, in order to achieve a variety of treatments simultaneously, or to enhance a single effect without causing severe side effects, a combination of herbs is conventionally used (19). In order to obtain a more effective remedy for the treatment of HCC other than exclusively using Paeonia lactiflora or Astragalus membranaceus, we combined these two herbs based on both traditional literature and the results of our previous study mentioned above. The standardized extract from Paeonia lactiflora and Astragalus membranaceus (PAE), with a standard ratio of 4:1 of these two plants, was mainly composed of TGP and the total astragalosides (20). Our previous animal studies have confirmed that PAE exhibited the most significant hepato-protective activity compared to exclusively using Paeonia lactiflora or Astragalus membranaceus (21). Our previous studies have shown that PAE had obvious protective effects on acute liver injury and chronic liver fibrosis induced by carbon tetrachloride (CCl4) $(20,22)$. These above studies have clearly demonstrated that PAE is an effective and therapeutic agent for chronic liver disease.

HCC is one of the main complications of chronic liver diseases. HCC mainly developed in the background of advanced liver fibrosis and cirrhosis through the malignant transformation of cirrhotic nodules and premalignant lesions (23). The usual multistep process of fibrosis-cirrhosis-HCC, has been recognized as the significant progresses of HCC (24). Previous research in our laboratory has evidenced that PAE possessed a distinct inhibitory effect on hepatic fibrosis. The data obtained from our previous studies created considerable interest in PAE as a therapeutic agent in HCC.

In the present study, to determine the effect of PAE on HCC cell proliferation, apoptosis, migration and invasion, in cultured human HCC cell line HepG2 and SMMC-7721 as a model system and we analysed the changes after treatment with PAE. The results of this study show that PAE revealed obviously pro-apoptosis and inhibitory effect on proliferation, migration and invasion of HCC cells. These data suggested that PAE may be a novel, efficient candidate agent for treatment of $\mathrm{HCC}$.

\section{Materials and methods}

Preparation of PAE. Radix Paeonia lactiflora and Radix Astragali were purchased from Hefei Heyitang Pharmacy, China. Voucher specimens (Pan 2009002 and Pan 2009013) were identified and deposited in relevant departments of Anhui College of Traditional Chinese Medicine. The sliced dried roots of Radix Paeonia lactiflora and Radix Astragali were mixed in the ratio of $4: 1$. The mixed roots $(25 \mathrm{~kg})$ were extracted two times with $70 \%$ aqueous ethanol, six times $1.5 \mathrm{~h}$ each time. Then, the extracts were combined and evaporated to dry powder under reduced pressure. The residue obtained from the combined extracts was dissolved in 81 of water. After filtration, the aqueous solution was extracted three times with 81 water-saturated $n$-butanol successively each time, which yielded dry powder after being combined and evaporated to dryness under reduced pressure. Then, $\sim 1 \mathrm{~kg}$ of the $n$-butanol extract was chromatographed on polystryrene resin (D101,
0.3-1.25 mm, Nankai Chemical Factory, Tianjin, China) with water, $10 \%$ ethanol and $70 \%$ ethanol, respectively. Portions of $70 \%$ ethanol were collected and evaporated to dryness under reduced pressure, which yielded $310 \mathrm{~g}$ of yellow dry powder. The result of chromatometry showed that the dry powder contained $74.7 \%$ total glycoside of paeony (TGP) and $20.6 \%$ astragalosides (ASTs). TGP consist mainly of paeoniflorin, oxypaeoniflorin, benzoyl paeoniflorin, albiflorin and lactiflorin (25). ASTs consist of astragaloside I-IV and aoyasapogenoside (20). Before treatment on HCC cells, PAE was suspended in phosphate-buffered saline (PBS).

Chemicals and reagent. MTT was purchased from Sigma Chemical Co. (St. Louis, MO, USA). Cell Apoptosis PI Detection Kit was provided by Nanjing KeyGen Biotech Co. Ltd. (Nanjing, China). Bcl-2, Bax and cleaved caspase-3 primary antibody were purchased from Santa Cruz Biotechnology (Santa Cruz, CA, USA). Dulbecco's modified Eagle's medium (DMEM) and fetal bovine serum (FBS) were obtained from

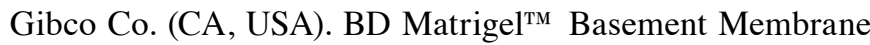
Matirx was purchased from BD Biosciences (Bedford, MA, USA). Other chemicals used in the experiment were analytical grade from commercial sources.

Cell culture. Human HCC cell line HepG2 and SMMC-7721 was purchased from Shanghai Cell Bank, Chinese Academy of Sciences (Shanghai, China), cultured in DMEM supplemented with $10 \%$ (v/v) FBS and $100 \mathrm{U} / \mathrm{ml}$ of penicillin and $100 \mu \mathrm{g} /$ $\mathrm{ml}$ of streptomycin. HepG2 and SMMC-7721 cells were maintained at $37^{\circ} \mathrm{C}$ in a $5 \% \mathrm{CO}_{2}$ atmosphere.

Cell proliferation assay. MTT assay was performed to determine the number of viable cells. The cells were cultured in 96-well plates at a density of $1 \times 10^{4}$ cells per well with fresh medium containing PAE at the various concentrations (12.5, $25,50,100$ and $200 \mathrm{mg} / \mathrm{l})$. After treatment for 24,48 or $72 \mathrm{~h}$, MTT solution was added $(20.0 \mu \mathrm{l} /$ well $)$ and then the plates were incubated at $37^{\circ} \mathrm{C}$ in $5 \% \mathrm{CO}_{2}$ for another $4 \mathrm{~h}$. The MTT-formazan product were dissolved in $150 \mu \mathrm{l}$ dimethyl sulfoxide (DMSO) per well. After $10 \mathrm{~min}$, the plates were read on BioTek Elxx808 microplate reader (Winooski, VT, USA) at $490 \mathrm{~nm}$.

Flow cytometry analysis. HepG2 and SMMC-7721 cells $\left(1 \times 10^{6}\right.$ cells per well) were plated in 6-well plates and treated with PAE $(12.5,25,50,100$ and $200 \mathrm{mg} / \mathrm{l})$ for $48 \mathrm{~h}$. After being harvested by trypsinization, the cells were washed twice with cold PBS and centrifuged. The cell pellet was resuspended in $1 \mathrm{ml}$ cold PBS and fixed in $9 \mathrm{ml}$ of $70 \%$ ethanol at $-20^{\circ} \mathrm{C}$ for $\geq 12 \mathrm{~h}$. RNase was added and the cells incubated at $37^{\circ} \mathrm{C}$ for $30 \mathrm{~min}$ then centrifuged and resuspended in $500 \mu \mathrm{l} \mathrm{PBS}$. Next, propidium iodide (PI) staining buffer was added, and left in the dark at room temperature for $30 \mathrm{~min}$. The cells were collected and apoptosis was examined by FC 500 (Beckman Coulter, Brea, CA, USA).

Immunocytochemistry analysis. Cells were seeded onto glass coverslips overnight. After incubation with various concentrations of PAE for $48 \mathrm{~h}$, the coverslips were washed twice with PBS and $4 \%$ paraformaldehyde solution was added for 
Table I. Effect of PAE on the proliferation of HepG2 cells $(\bar{x} \pm s, \mathrm{n}=8)$.

\begin{tabular}{|c|c|c|c|c|c|c|c|}
\hline \multirow[b]{2}{*}{ Group } & \multirow[b]{2}{*}{$\begin{array}{c}\text { Concentration } \\
(\mathrm{mg} / \mathrm{l})\end{array}$} & \multicolumn{2}{|c|}{$24 \mathrm{~h}$} & \multicolumn{2}{|c|}{$48 \mathrm{~h}$} & \multicolumn{2}{|c|}{$72 \mathrm{~h}$} \\
\hline & & $\mathrm{A}_{490 \mathrm{~nm}}$ & $\begin{array}{l}\text { Inhibitory } \\
\text { rate }(\%)\end{array}$ & $\mathrm{A}_{490 \mathrm{~nm}}$ & $\begin{array}{c}\text { Inhibitory } \\
\text { rate }(\%)\end{array}$ & $\mathrm{A}_{490 \mathrm{~nm}}$ & $\begin{array}{c}\text { Inhibitory } \\
\text { rate }(\%)\end{array}$ \\
\hline Control & - & $0.451 \pm 0.025$ & - & $0.530 \pm 0.019$ & - & $0.937 \pm 0.012$ & - \\
\hline \multirow[t]{5}{*}{ PAE } & 12.5 & $0.430 \pm 0.017$ & 4.66 & $0.496 \pm 0.035$ & 6.39 & $0.824 \pm 0.050$ & 11.96 \\
\hline & 25 & $0.413 \pm 0.042$ & 8.39 & $0.473 \pm 0.021$ & 10.66 & $0.738 \pm 0.033^{\mathrm{a}}$ & 21.19 \\
\hline & 50 & $0.372 \pm 0.040^{\mathrm{a}}$ & 17.52 & $0.399 \pm 0.051^{\mathrm{b}}$ & 24.53 & $0.724 \pm 0.029^{\mathrm{a}}$ & 22.73 \\
\hline & 100 & $0.282 \pm 0.026^{\mathrm{b}}$ & 37.49 & $0.309 \pm 0.023^{b}$ & 41.69 & $0.559 \pm 0.015^{\mathrm{b}}$ & 40.32 \\
\hline & 200 & $0.203 \pm 0.022^{b}$ & 55.03 & $0.195 \pm 0.048^{\mathrm{b}}$ & 63.21 & $0.445 \pm 0.043^{b}$ & 52.56 \\
\hline
\end{tabular}

${ }^{\mathrm{a}} \mathrm{P}<0.05 ;{ }^{\mathrm{b}} \mathrm{P}<0.01$ compared with control group.

$30 \mathrm{~min}$ at room temperature. Immunocytochemistry staining for Bcl-2 and Bax was performed according to the manufacturer's instructions (Zhongshan Jinqiao, Beijing, China). PBS was used as a negative control instead of the primary antibody. The results were quantitatively analyzed by Olympus BX53F video microscope (Olympus, Tokyo, Japan) and the Image-Pro Plus software (Media Cybernetics, Rockville, MD, USA) in five random fields per slide. The relative intensity of Bcl-2 and Bax were reflected by optical density value.

Western blot analysis. HepG2 and SMMC-7721 cells were treated with PAE at various gradient concentrations. Then protein was extracted from cells in RIPA lysis buffer [50 mmol/1 Tris-HCl, pH 7.4, $150 \mathrm{mmol} / 1 \mathrm{NaCl}, 10 \mathrm{mmol} / 1$ phenylmethylsulfonyl fluoride (PMSF), $1 \mathrm{mmol} / 1$ ethylene diamine tetraacetic acid (EDTA), $0.1 \%$ sodium dodecyl sulfate (SDS), $1 \%$ Triton $\mathrm{X}-100,1 \%$ sodium deoxycholate]. The protein concentration was determined with the Bradford assay. A protein sample was mixed with the $5 \mathrm{X}$ sample buffer $(4: 1)$ (Bio-Rad, Hercules, CA, USA) and heated in boiling water for $10 \mathrm{~min}$. The proteins were resolved by SDS-PAGE, transferred to a polyvinylidene fluoride (PVDF) membrane (Millipore, Bedford, MA, USA). After blocking with 5\% non-fat milk (blocking solution) at room temperature, the membrane was incubated with primary antibodies diluted in blocking solution overnight at $4^{\circ} \mathrm{C}$. After washing the blot in TBST three times, the horseradish peroxidase (HRP)-conjugated secondary antibodies were applied for $2 \mathrm{~h}$ at room temperature. After extensive washing in TBST, immunodetection was visualized by enhanced chemiluminescence (Pierce, Rockford, IL, USA) using hydrogen peroxide and luminol as substrates. Autoradiographs were scanned using Image Quant LAS 4000 mini (GE Healthcare Bio-Sciences AB, Uppsala, Sweden). The density of the specific bands was quantified using ImageJ software (National Institutes of Health, Bethesda, MD, USA).

Wound healing assay. HepG2 and SMMC-7721 cells were seeded into 24 -well plates, at $80-90 \%$ confluency, the cell monolayer was wounded with a $200 \mu$ l-pipette. After washing with PBS three times to remove cell debris, the remaining cells were treated with $12.5,25,50,100$ or $200 \mathrm{mg} / 1 \mathrm{PAE}$ and then images were captured by microscope at 0 and $24 \mathrm{~h}$ after treatment. Cell motility was evaluated according to the following formula: Cell motility $=($ distance $24 \mathrm{~h}$ - distance $0 \mathrm{~h}) /$ distance $0 \mathrm{~h}$.

Matrigel Transwell assay. The invasion assay was performed using Transwell co-culture chambers ( 24 wells, $8-\mu$ m pore size, Costar, Corning Inc., NY, USA). In brief, upper inserts were coated with $30 \mu \mathrm{l}$ Matrigel (1:4 in dilution; BD Biosciences) and then to set for $0.5 \mathrm{~h}$ at $37^{\circ} \mathrm{C}$. SMMC-7721 cells $\left(5 \times 10^{5}\right)$ were resuspended in $100 \mu \mathrm{l}$ serum-free DMEM medium containing $0.1 \%$ BSA and were added into the upper insert, while in the lower chamber containing DMEM $(600 \mu \mathrm{l})$ with $10 \%$ FBS as a chemoattractant. Then DMEM medium free of FBS added 12.5, 25, 50, 100 or $200 \mathrm{mg} / \mathrm{l}$ PAE in the upper chamber. After $36-\mathrm{h}$ incubation at $37^{\circ} \mathrm{C}$, the SMMC-7721 cells in the upper chambers were scraped off carefully with swabs; the invaded cells were fixed and stained with $0.1 \%$ crystal violet. Following air-drying, the invaded SMMC-7721 cells were counted in 5 random visual fields $(\mathrm{x} 400)$ for each insert.

Statistical analysis. Data are expressed as the means \pm SD and statistical analysis was performed using one-way ANOVA. All statistical analyses were performed with the statistical package SPSS 13.0 (SPSS Inc, Chicago, IL, USA). A level of $\mathrm{P}<0.05$ was considered statistically significant.

\section{Results}

Inhibitory activity of PAE on hepatoma cell proliferation. To determine the role of PAE in the proliferation of HepG2 and SMMC-7721 cells, cells were incubated with various concentrations of PAE for 24, 48 or $72 \mathrm{~h}$. As shown in Table I, the inhibitory rate of PAE $(12.5,25,50,100$ and $200 \mathrm{mg} / \mathrm{l})$ on HepG2 cells was 6.39, 10.66, 24.53, 41.69 and $63.21 \%$ and the inhibitory rate of PAE on SMMC-7721 cells was 2.81, 13.61, $33.66,41.73$ and $68.33 \%$ (Table II) after treatment for $48 \mathrm{~h}$. The MTT experiment confirmed that hepatoma cells were sensitive to PAE and PAE displayed an inhibitory effect on the proliferation of human hepatoma cells.

PAE induces apoptosis of HepG2 and SMMC-7721 cells. To determine whether PAE induced apoptosis of HepG2 and 
Table II. Effect of PAE on the proliferation of SMMC-7721 cells $(\bar{x} \pm s, \mathrm{n}=8)$.

\begin{tabular}{|c|c|c|c|c|c|c|c|}
\hline \multirow[b]{2}{*}{ Group } & \multirow[b]{2}{*}{$\begin{array}{l}\text { Concentration } \\
(\mathrm{mg} / \mathrm{l})\end{array}$} & \multicolumn{2}{|c|}{$24 \mathrm{~h}$} & \multicolumn{2}{|c|}{$48 \mathrm{~h}$} & \multicolumn{2}{|c|}{$72 \mathrm{~h}$} \\
\hline & & $\mathrm{A}_{490 \mathrm{~nm}}$ & $\begin{array}{l}\text { Inhibitory } \\
\text { rate }(\%)\end{array}$ & $\mathrm{A}_{490 \mathrm{~nm}}$ & $\begin{array}{c}\text { Inhibitory } \\
\text { rate }(\%)\end{array}$ & $\mathrm{A}_{490 \mathrm{~nm}}$ & $\begin{array}{c}\text { Inhibitory } \\
\text { rate }(\%)\end{array}$ \\
\hline Control & - & $0.467 \pm 0.022$ & - & $0.498 \pm 0.021$ & - & $0.992 \pm 0.052$ & - \\
\hline \multirow[t]{5}{*}{ PAE } & 12.5 & $0.437 \pm 0.027$ & 6.28 & $0.484 \pm 0.047$ & 2.81 & $0.975 \pm 0.050$ & 1.65 \\
\hline & 25 & $0.419 \pm 0.035$ & 10.27 & $0.430 \pm 0.042^{\mathrm{a}}$ & 13.61 & $0.843 \pm 0.048^{a}$ & 14.96 \\
\hline & 50 & $0.389 \pm 0.027^{\mathrm{a}}$ & 16.64 & $0.330 \pm 0.030^{\mathrm{b}}$ & 33.66 & $0.672 \pm 0.015^{\mathrm{b}}$ & 32.23 \\
\hline & 100 & $0.248 \pm 0.032^{\mathrm{b}}$ & 46.94 & $0.290 \pm 0.036^{\mathrm{b}}$ & 41.73 & $0.612 \pm 0.041^{\mathrm{b}}$ & 38.26 \\
\hline & 200 & $0.170 \pm 0.022^{\mathrm{b}}$ & 63.68 & $0.158 \pm 0.025^{\mathrm{b}}$ & 68.33 & $0.492 \pm 0.033^{\mathrm{b}}$ & 50.41 \\
\hline
\end{tabular}

${ }^{\mathrm{a}} \mathrm{P}<0.05 ;{ }^{\mathrm{b}} \mathrm{P}<0.01$ compared with control group.

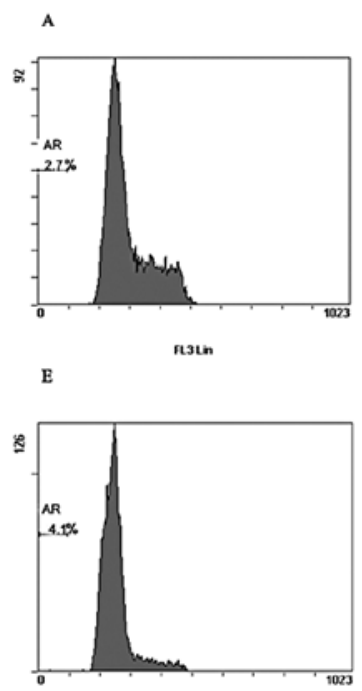

คงเก
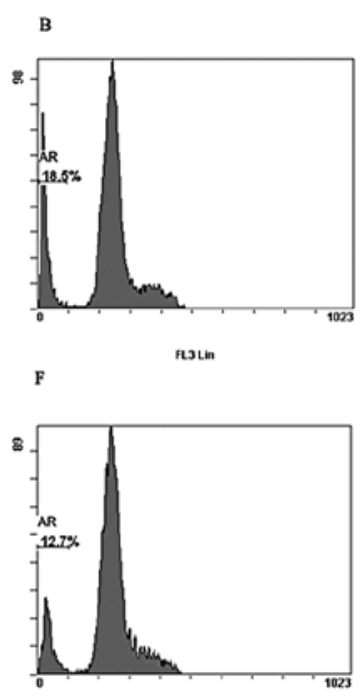

flutim

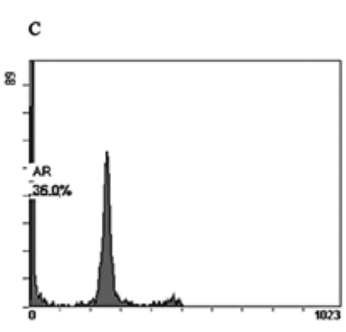

G

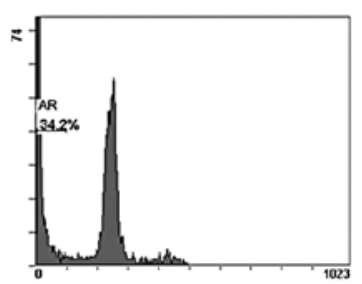

fluth

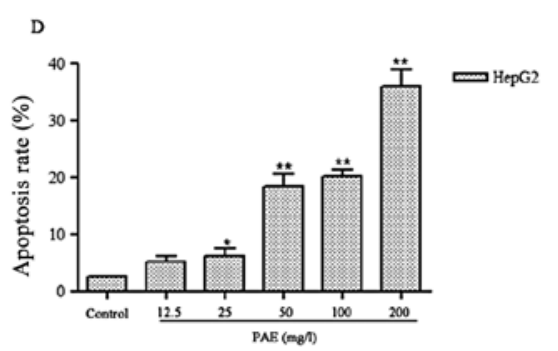

$\mathrm{H}$

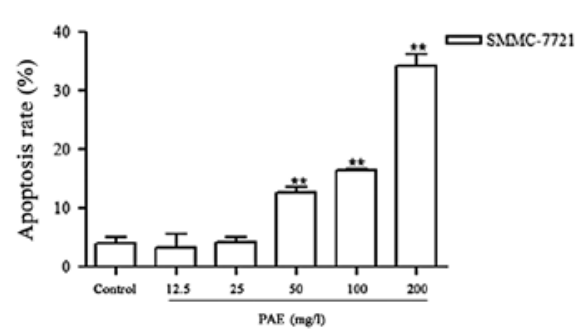

Figure 1. Effect of PAE on apoptosis of HepG2 cells and SMMC-7721 cells. AR, apoptosis rate. HepG2 (A) and SMMC-7721 cells (E) without any PAE treatment were used as the control group. HepG2 (B and C) and SMMC-7721 (F and G) cells treated with PAE (50 and 200 mg/l). (D and H) The semi-quantitative analysis showed the changes in the apoptosis rate of HepG2 cells and SMMC-7721 cells. The data are expressed as the mean \pm SD in the three independent experiments. ${ }^{*} \mathrm{P}<0.05,{ }^{* *} \mathrm{P}<0.01$ compared with the control group.

SMMC-7721 cells, a Cell Apoptosis PI Detection kit was applied by flow cytometry. As shown in Fig. 1, the percentage of apoptotic cells increased from $2.7 \%$ in control treated cells to $36.0 \%$ in PAE $200 \mathrm{mg} / 1$ treatment group of HepG2 cells (Fig. 1C). Similar results were observed in SMMC-7721 cells. PAE $200 \mathrm{mg} / \mathrm{l}$ treatment increased the percentage of apoptotic cells by $34.2 \%$ in SMMC-7721 cells (Fig. 1G). These results showed that PAE significantly induced apoptosis of hepatoma cells.

PAE modulates the expression of Bcl-2 and Bax in HepG2 and SMMC-7721 cells. To identify a possible mechanism by which PAE induced apoptosis in hepatoma cells, immunocytochemistry was performed. Our data demonstrated that morphological changes occurred during PAE exposure in HepG2 and SMMC-7721 cells (Figs. 2 and 3). PAE exposureinduced apoptosis in the hepatoma cells was identified by cell shrinkage and pyknosis. Cell shrinkage leads to smaller cells and the cytoplasm was dense and the organelles were more tightly packed. In addition, Bax staining was observed with PAE treatment at 12.5, 25, 50, 100 and $200 \mathrm{mg} / \mathrm{l}$. Bax expression was the most prominent after treatment with $200 \mathrm{mg} / \mathrm{l}$ PAE. The expression of Bax in the control group presented weak staining in the cytoplasm (Figs. 2A and 3A). However, the expression of Bcl-2 presented strong dark brown staining in the control group (Figs. 2B and 3B). With concentrations of 50, 100 and $200 \mathrm{mg} / \mathrm{l} \mathrm{PAE}$ the expression of Bcl-2 presented weak brown staining or lack of staining.

Consistent with immunocytochemistry results, western blot analysis showed decreased expression of Bcl-2 in PAE-treated hepatoma cells compared to control group. PAE at concentrations of 50, 100 and $200 \mathrm{mg} / 1$ markedly inhibited Bcl-2 expression. However, the expression of Bax was elevated in various concentrations of PAE (50, 100 and 
A
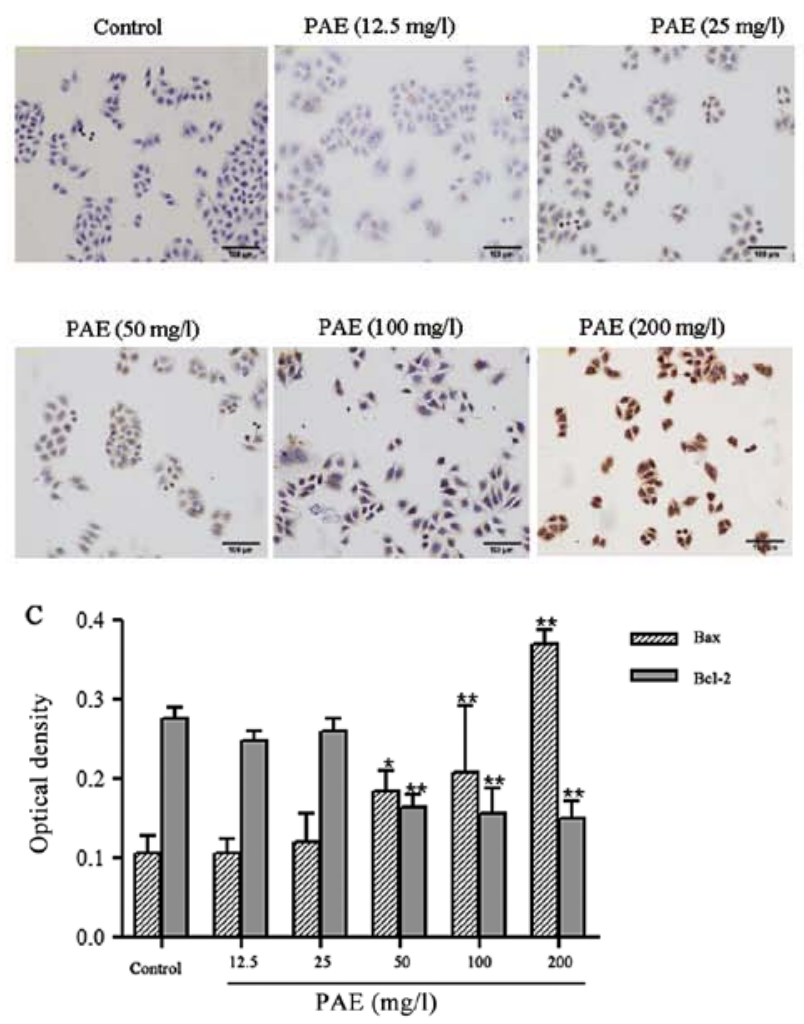

A
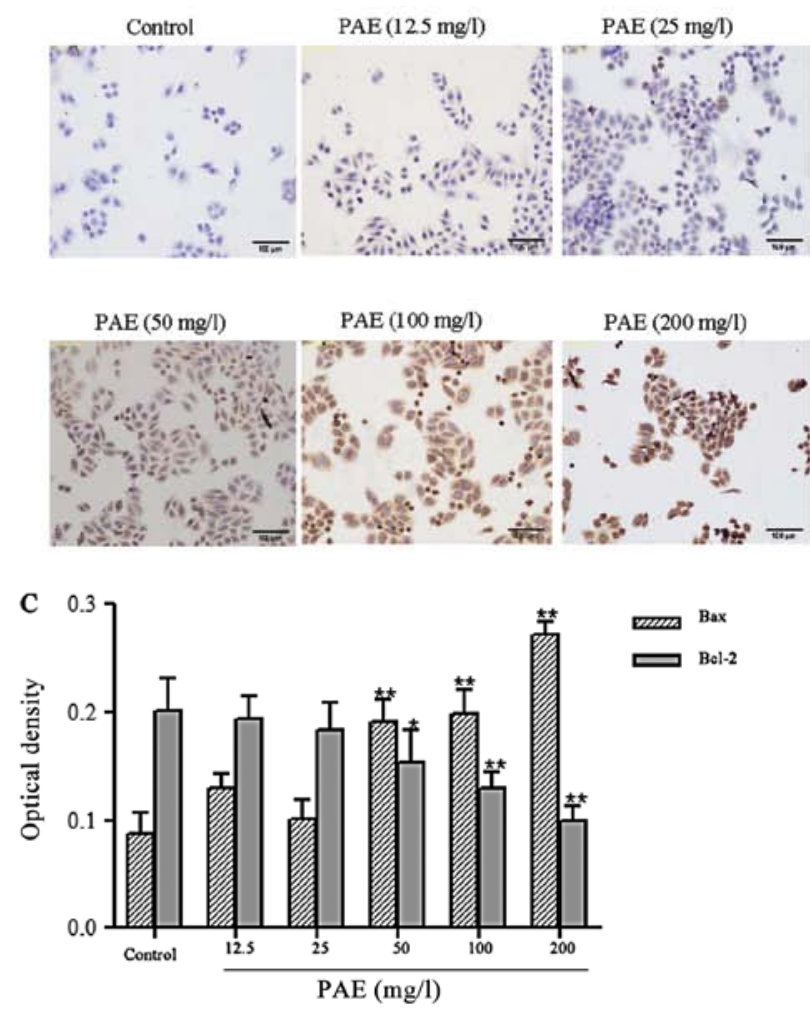

$200 \mathrm{mg} / \mathrm{l}$ ) treated cells compared to the untreated control cells. As shown in Fig. 4C, the Bax to Bcl-2 ratio was significantly increased in the PAE treated cells compared to the control
B
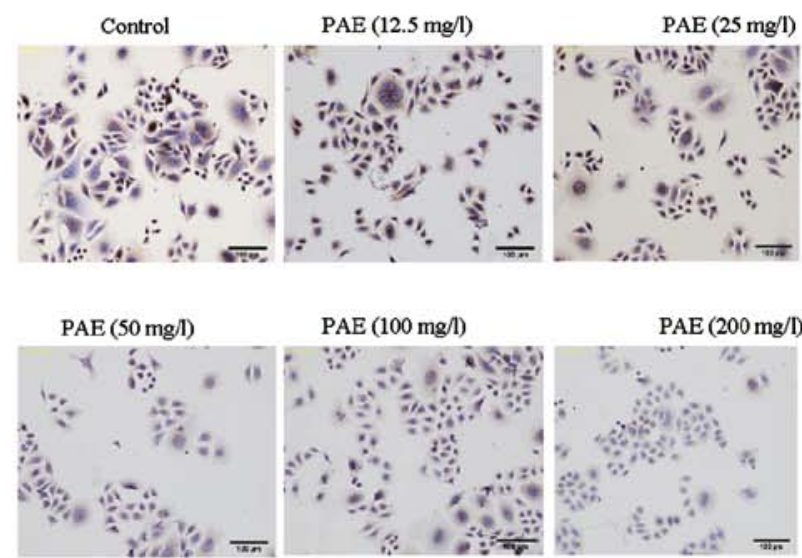

Figure 2. The expression of Bax and Bcl-2 in immunohistochemistry-stained HepG2 cells. Effects of PAE on the expression of Bax (A) and Bcl-2 (B) in HepG2 cells. Cells without any PAE treatment were used as the baseline control. (C) The semi-quantitative analysis of Bax and Bcl-2 expression in HepG2 cells. The data are expressed as the mean \pm SD in the three independent experiments. ${ }^{*} \mathrm{P}<0.05,{ }^{* *} \mathrm{P}<0.01$ compared with the control group.

B
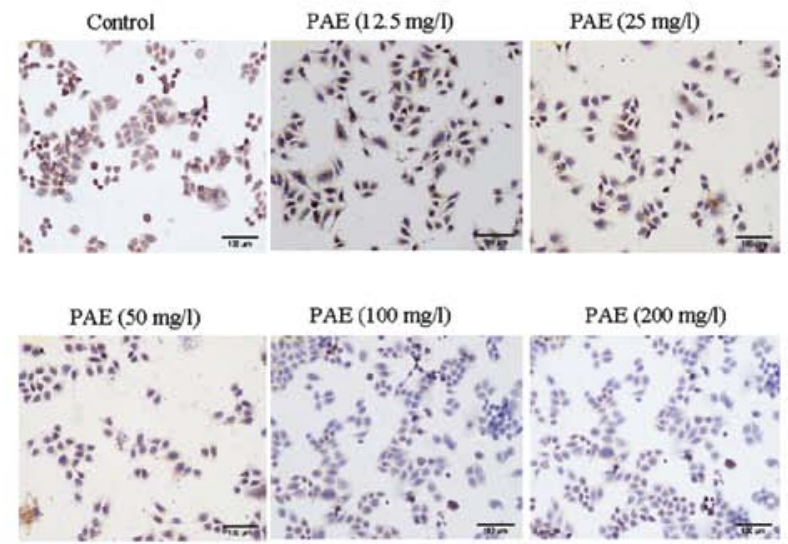

Figure 3. The expression of Bax and Bcl-2 in immunohistochemistry-stained SMMC-7721 cells. Effect of PAE on the expression of Bax (A) and Bcl-2 (B) in SMMC-7721 cells. Cells without any PAE treatment were used as the baseline control. (C) The semi-quantitative analysis of Bax and Bcl-2 expression SMMC-7721 cells. The data are expressed as the mean \pm SD in the three independent experiments. ${ }^{*} \mathrm{P}<0.05,{ }^{* *} \mathrm{P}<0.01$ compared with the control group.

group. The results indicate that PAE induces apoptosis in HepG2 and SMMC-7721 cells mainly through regulating the Bcl-2 family. 
A

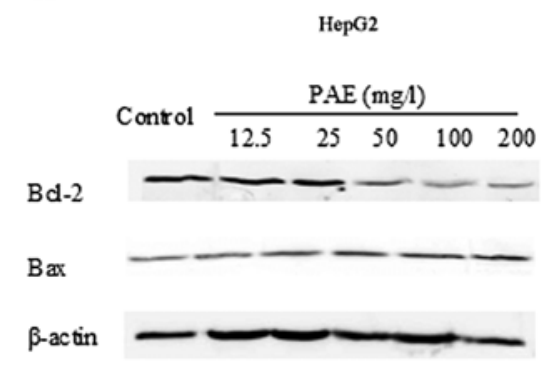

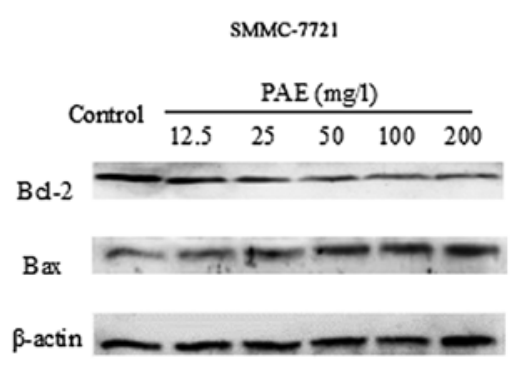

C

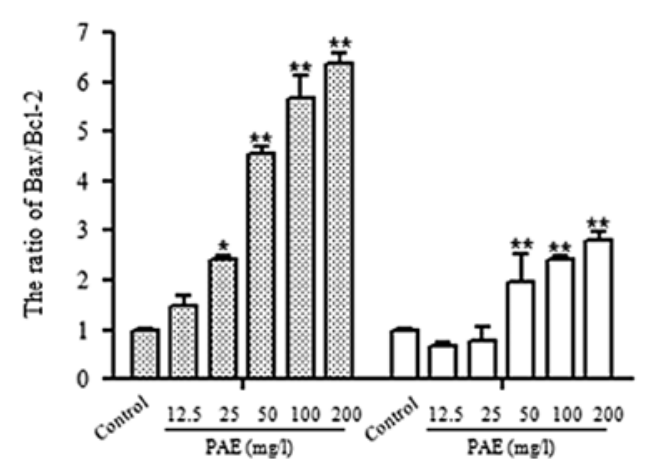

Figure 4. Effects of PAE on Bax and Bcl-2 expression in HepG2 and SMMC-7721 cells. Western blot images of HepG2 (A) and SMMC-7721 (B) cells. (C) The ratio of Bax to Bcl-2 in HepG2 and SMMC-7721 cells increased in PAE-treated cells compared with the cells without any treatment. The data from three independent experiments are shown as the mean $\pm \mathrm{SD}$. ${ }^{*} \mathrm{P}<0.05,{ }^{* * *} \mathrm{P}<0.01$ compared with the control group.

A

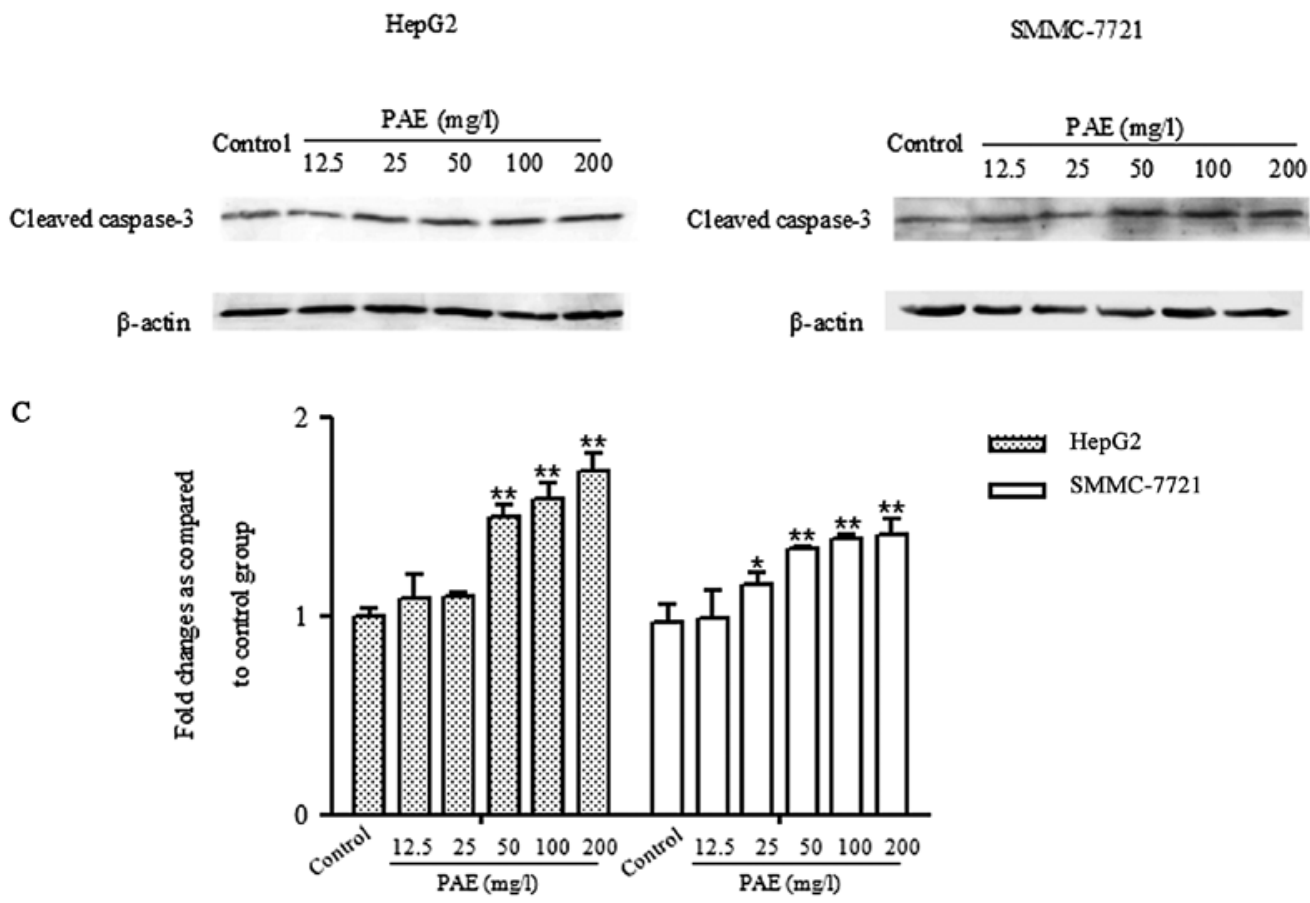

Figure 5. Effects of PAE on cleaved caspase-3 expression in HepG2 and SMMC-7721 cells. Western blot images of HepG2 (A) and SMMC-7721 (B) cells. (C) The semi-quantitative analysis of cleaved caspase-3. The data from three independent experiments are shown as the mean $\pm \mathrm{SD}$. ${ }^{*} \mathrm{P}<0.05,{ }^{* * *} \mathrm{P}<0.01$ compared with the control group.

PAE increases caspase-3 activation in HepG2 and SMMC7721 cells. To determine whether PAE effects the activation of caspase-3 in HepG2 and SMMC-7721 cells, the activation of caspase- 3 was quantified through western blotting. As shown in Fig. 5, low levels of cleaved caspase-3 were observed in control treated cells. Treatment with various concentrations 
$\mathbf{A}$
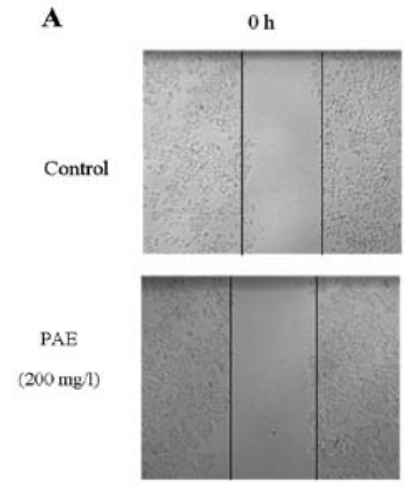

C
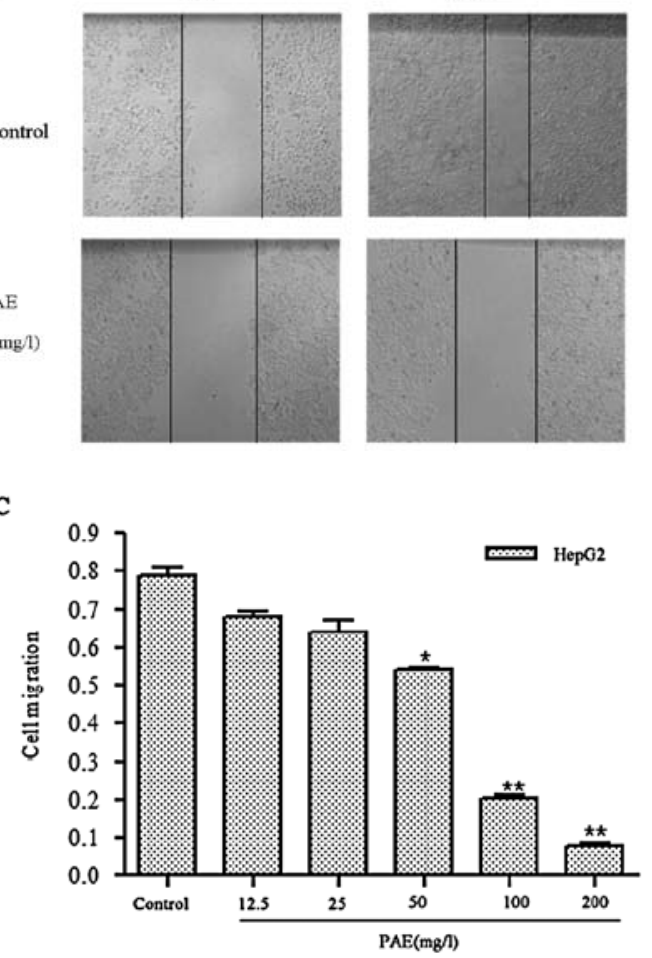

B

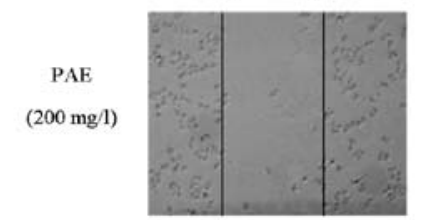

D

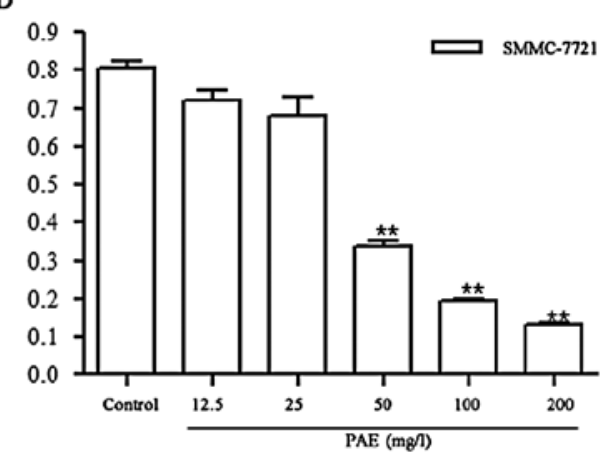

Figure 6. Effects of PAE on the migration of HepG2 cells and SMMC-7721 cells. Wound-healing analysis of HepG2 and SMMC-7721 cell migration for 0 and $24 \mathrm{~h}$, cells were treated with 0-200 mg/l PAE. (A) Images of HepG2 cells after treatment of PAE $200 \mathrm{mg} / \mathrm{l}$ at 0 and $24 \mathrm{~h}$ compared with control group. (B) Images of SMMC-7721 cells after treatment of PAE $200 \mathrm{mg} / 1$ at 0 and $24 \mathrm{~h}$ compared with control group. (C) Quantitative analysis of various concentrations of PAE treated HepG2 cells. (D) Quantitative analysis of PAE treated SMMC-7721 cells. The data from three independent experiments are shown as the mean $\pm \mathrm{SD} .{ }^{*} \mathrm{P}<0.05,{ }^{* *} \mathrm{P}<0.01$ compared with the control group.

A
Control

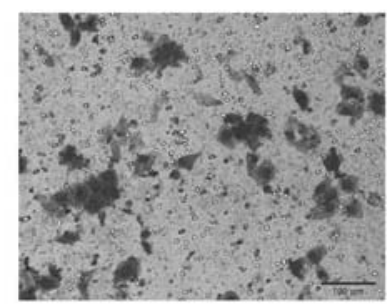

B

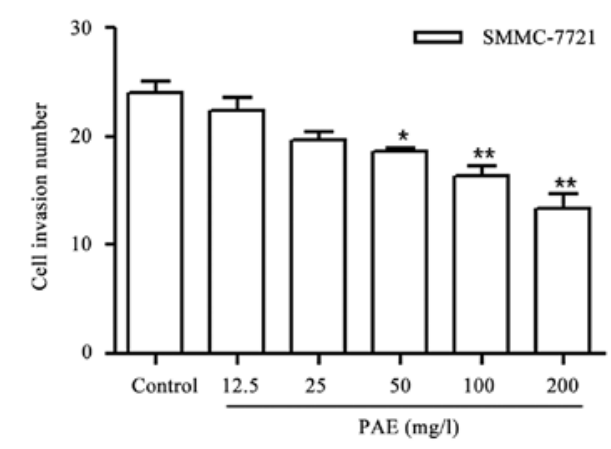

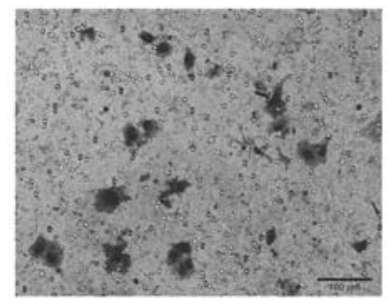

$\operatorname{PAE}(200 \mathrm{mg} / 1)$

(5)

at 0 at 0 and $24 \mathrm{~h}$. The results showed that PAE (50, 100 and $200 \mathrm{mg} / \mathrm{l})$ significantly suppressed the migration ability of HepG2 cells and SMMC-7721 cells (Fig. 6). As shown in Fig. 7A, transwell invasion assay revealed a significant reduction in invasion of SMMC-7721 cells treated with PAE $200 \mathrm{mg} / 1$ in comparison to control cells. These two observations emphasized the inhibitive role of PAE in HCC cell migration and invasion.

\section{Discussion}

Despite many years of intensive research, $\mathrm{HCC}$ remains a devastating malignancy (26). Conventional chemotherapy is one of the common strategies for HCC treatment $(27,28)$. Although chemotherapeutic drugs have been administered to patients with HCC, the curative effect was often accompanied by various side effects (6). Therefore, the development of new drugs with the ability to directly inhibit cancer cell proliferation and induce cell apoptosis for the treatment of HCC is 
urgent needed. Recently, chemotherapy and other intervention strategies using extracts of Chinese herbal medicines have emerged as a promising alternative option to improve the quality of life for HCC patients (5,29-31). For example, swainsonine (an extract from Sphaerophysa salsula or Astragalus membranaceus) and paeoniflorin (extracted from the root of Paeonia lactiflora), have been confirmed to inhibit cell growth, induce apoptosis and potentiate the cytotoxic effect on HCC cells and cervical cancer cells $(12,32,33)$. PAE was designed according to the principles of Chinese traditional treatment on liver disease, as well as applying with the contemporary extraction and purification technology (20). PAE in vivo was confirmed to exhibit obvious hepatoprotective effects on the acute liver injury and chronic liver fibrosis $(20,22)$. Moreover, in vitro studies indicated the primary mechanisms of its anti-liver injury effects may be associated with inhibiting proliferation of hepatic stellate cells and MAPK activation (34). In addition, these previous results have proved that PAE exerted conspicuously higher efficiency than the use of the extracts TGP and total astragalosides exclusively.

It has been well accepted that the development process of HCC mostly connected with the chronic liver disease, such as liver fibrosis (24), and the usual pattern of fibrosis-cirrhosis$\mathrm{HCC}$, has been revealed for many years. Combined with the previous studies, we hypothesized that PAE could also affect the follow-up development of fibrosis, the liver cancer. Therefore, the present study was designed to firstly investigate whether PAE inhibits proliferation or induces apoptosis. Our proliferation and flow cytometry assays revealed that PAE significantly inhibits proliferation and induces apoptosis of HepG2 and SMMC-7721 cells. Then, to further determine the possible mechanisms of pro-proliferation and anti-apoptosis which were induced by PAE, the expression of the Bcl-2 family proteins and caspase-3 which are acknowledged apoptosis-related regulators, was examined. The immunocytochemistry analysis and western blotting results showed decreased expression of Bcl-2 and elevated levels of Bax and caspase-3 in HepG2 and SMMC-7721 cells treated with PAE compared with the control groups. These results suggest that PAE may mediate apoptosis by modulating the expression of Bcl-2 and caspase family proteins.

Apoptosis is a physiological cell death program that is a vital pathway in maintaining tissue homeostasis (35). Several factors contribute to apoptosis, but the pivotal ones are classified into two main families of proteins including $\mathrm{Bcl}-2$ family and caspase enzymes (36). The Bcl-2 gene (B-cell lymphoma/leukemia-2) is one of members of Bcl-2 family, which is closely related to apoptosis (37). Certain Bcl-2 family proteins have been recognized as a critical checkpoint within apoptotic pathways (38). The Bcl-2 family is composed of a number of genes, incuding the anti-apoptotic and pro-apoptotic members, such as Bcl-2 and Bax, which play critical roles in the control of mitochondrial integrity, have been considered to the crucial switch of the apoptotic process $(39,40)$. A large body of research indicates that elevated expression of Bcl-2 inhibits apoptosis in various cell types, inducing cancer cells (12). The excessive expression of Bcl-2 leads to the resistance of cells against cellular toxins, which may connect a mutual pathway or crosstalk in the signal transduction pathway regulated by Bcl-2 (41).
The results of our present studies showed by immunocytochemistry analysis and western blotting indicated that PAE reduced the expression of Bcl-2 and enhanced the expression of Bax, comparing to the non-treated groups, along with an increased expression of caspase-3.

Caspases are known for playing an important role in the execution of apoptosis, which frequently activates death protease, catalyzing the specific cleavage of many pivotal cellular proteins (42). During apoptosis, active caspase-3 leads to a self-amplifying cascade of proteolysis and cleavage of many effect or proteins. Caspase-3 is essential for cellular DNA damage and apoptosis (43). Certain studies have shown that overexpression of Bcl-2 prevents the mitochondrial release of cyt $c$, thereby inhibiting the activation of caspases cascade and apoptosis (44). The increase in caspase-3 activation is synchronized with an increased expression of pro-apoptotic protein Bax and a decreased expression of anti-apoptotic protein $\mathrm{Bcl}-2$ (45). The western blot analysis in this study demonstrated that PAE enhanced the expression of cleaved caspase- 3 both in HepG2 and SMMC-7721 cell lines. In agreement with the data of the Bcl-2 family, our study indicated PAE may induce apoptosis through the mechanisms of modulating the Bcl-2 family protein and the caspase-3 activity.

Due to invasion and intrahepatic metastasis, the prognosis for patients with $\mathrm{HCC}$ is poor. Invasive growth and metastatic potential is the basic reason which leads to stubborn and refractory liver cancer (46). The essential steps for metastases, invasion and migration require the action of tumor-associated proteases that dissolve the surrounding tumor matrix and basement membrane (47). Therefore, wound healing and Matrigel transwell assays were designed to detect the migratory and invasive abilities of HepG2 and SMMC-7721 cells after treatment with PAE. The results showed that PAE significantly inhibited the migration and invasion of HCC cells and provided new insights into the potential use of PAE in controlling $\mathrm{HCC}$ invasion and metastasis.

In conclusion, our study demonstrated that PAE caused significant inhibition of cell proliferation, migration and invasion and pro-apoptotic effect in HCC cells. Our data also indicated the antitumor effect of PAE is due to the upregulation of the Bax-to-Bcl-2 ratio, as well as activation of the caspase-dependent signaling pathway. As far as we know, the study on association between PAE and HCC cell proliferation, migration, invasion and apoptosis has not been investigated before. However, further studies are required to elucidate the detailed mechanisms of PAE effect on apoptosis and metastases. In view of the present results, PAE may be considered as a potential therapeutic candidate in treatment of HCC.

\section{Acknowledgements}

This study was supported by grants from the Specialized Research Fund for the Doctoral Program of Higher Education of China (no. 20113420120002), the Natural Science Foundation of the Higher Education Institutions of Anhui Province (no. KJ2012A153), the Natural Science Foundation of China (no. 81300332). The authors acknowledge the help of the staff members of the Institute of Clinical Pharmacology, Anhui Medical University, in conducting the study. 


\section{References}

1. El-Serag HB and Rudolph KL: Hepatocellular carcinoma: epidemiology and molecular carcinogenesis. Gastroenterology 132 2557-2576, 2007

2. Yang JD and Roberts LR: Hepatocellular carcinoma: a global view. Nat Rev Gastroenterol Hepatol 7: 448-458, 2010.

3. He J, Gu D and Wu X, et al: Major causes of death among men and women in China. N Engl J Med 353: 1124-1134, 2005.

4. Villanueva A and Llovet JM: Targeted therapies for hepatocellular carcinoma. Gastroenterology 140: 1410-1426, 2011.

5. Ma L, Wen S, Zhan Y, He Y, Liu X and Jiang J: Anticancer effects of the Chinese medicine matrine on murine hepatocellular carcinoma cells. Planta Med 74: 245-251, 2008.

6. Wang S, Zheng Z, Weng Y, et al: Angiogenesis and anti-angiogenesis activity of Chinese medicinal herbal extracts. Life Sci 74 2467-2478, 2004.

7. Dai LM, Chen MZ and Xu SY: Protective effects of total glucocides of paeony on experimental hepatitis. Chinese Pharmacol Bull 9: 449-453, 1993.

8. Wang H, Wei W, Wang NP, et al: Effects of total glucosides of peony on immunological hepatic fibrosis in rats. World J Gastroenterol 11: 2124-2129, 2005

9. Zheng YQ and Wei W: Total glucosides of paeony suppresses adjuvant arthritis in rats and intervenes cytokine-signaling between different types of synoviocytes. Int Immunopharmaco 5: 1560-1573, 2005.

10. Wang SH, Wei W, Xu DJ and Cui Y: Proliferation inhibition and mechanism of TGP on HepG2 cell in vitro. Acta Universitatis Medicinalis Anhui 41: 547-549, 2006.

11. Wang SH, Wei W, Xu DJ, Xu SP and Cui Y: Proliferation inhibition of TGP on SMMC-7721 Cell in vitro. Anhui Med Pharmaceut J 10: 8-9, 2006.

12. Zhang L and Zhang S: Modulating Bcl-2 family proteins and caspase-3 in induction of apoptosis by paeoniflorin in human cervical cancer cells. Phytother Res 25: 1551-1557, 2011.

13. Wu H, Li W, Wang T, Shu Y and Liu P: Paeoniflorin suppress NF-kappaB activation through modulation of I kappaB alpha and enhances 5-fluorouracil-induced apoptosis in human gastric carcinoma cells. Biomed Pharmacother 62: 659-666, 2008.

14. Tsuboi H, Hossain K, Akhand AA, et al: Paeoniflorin induces apoptosis of lymphocytes through a redox-linked mechanism. J Cell Biochem 93: 162-172, 2004.

15. Zhang Q, Gao WY and Man SL: Chemical composition and pharmacological activities of astragali radix. Zhongguo Zhong Yao Za Zhi 37: 3203-3207, 2012 (In Chinese).

16. Law PC, Auyeung KK, Chan LY and Ko JK: Astragalus saponins downregulate vascular endothelial growth factor under cobalt chloride-stimulated hypoxia in colon cancer cells. BMC Complement Altern Med 12: 160, 2012.

17. Hu XY, Xia RX, Cheng CB, et al: Mechanism of apoptosis in human leukemia NB4 cells induced by total astragalosides. Zhonghua Zhong Liu Za Zhi 33: 345-348, 2011 (In Chinese).

18. Liu X, Yang Y, Zhang X, et al: Compound Astragalus and Salvia miltiorrhiza extract inhibits cell invasion by modulating transforming growth factor-beta/Smad in HepG2 cell. J Gastroenterol Hepatol 25: 420-426, 2010

19. Nishiyama N, Wang YL and Saito H: Beneficial effects of $\mathrm{S}-113 \mathrm{~m}$, a novel herbal prescription, on learning impairment model in mice. Biol Pharm Bull 18: 1498-1503, 1995.

20. Sun WY, Wei W, Wu L, Gui SY and Wang H: Effects and mechanisms of extract from Paeonia lactiflora and Astragalus membranaceus on liver fibrosis induced by carbon tetrachloride in rats. J Ethnopharmacol 112: 514-523, 2007.

21. Shao FR, Wei W, Liu H, et al: Comparison of protective effect of Shaoqiduogan extracted by different methods on immunological liver injury in mice. J Anhui TCM College 26: 21-24, 2007.

22. Sun WY, Wei W, Gui SY, Wu L and Wang H: Protective effect of extract from Paeonia lactiflora and Astragalus membranaceus against liver injury induced by bacillus Calmette-Guerin and lipopolysaccharide in mice. Basic Clin Pharmacol Toxicol 103 $143-149,2008$

23. Colombo M, de Franchis R, Del Ninno E, et al: Hepatocellular carcinoma in Italian patients with cirrhosis. N Engl J Med 325: 675-680, 1991.

24. Paradis V: Histopathology of hepatocellular carcinoma. Recent Results Cancer Res 190: 21-32, 2013.
25. Zhang XY and Wang J: A study on the chemical constituents of Paeonia lactiflora Pall. J Shenyang Pharmaceutl Univ 18: 30-32, 2001.

26. Rahbari NN, Mehrabi A, Mollberg NM, et al: Hepatocellular carcinoma: current management and perspectives for the future. Ann Surg 253: 453-469, 2011.

27. Alves RC, Alves D, Guz B, et al: Advanced hepatocellular carcinoma. Review of targeted molecular drugs. Ann Hepatol 10: 21-27, 2011

28. Yu YL, Lu Y, Tang X and Cui FD: Formulation, preparation and evaluation of an intravenous emulsion containing Brucea javanica oil and Coix Seed oil for antitumor application. Biol Pharm Bull 31: 673-680, 2008

29. Li WY, Chiu LC, Lam WS, et al: Ethyl acetate extract of Chinese medicinal herb Sarcandra glabra induces growth inhibition on human leukemic HL-60 cells, associated with cell cycle arrest and up-regulation of pro-apoptotic Bax/Bcl-2 ratio. Oncol Rep 17: 425-431, 2007

30. Luqman S and Pezzuto JM: NFkappaB: a promising target for natural products in cancer chemoprevention. Phytother Res 24: 949-963, 2010

31. Karikas GA: Anticancer and chemopreventing natural products: some biochemical and therapeutic aspects. J BUON 15: 627-638, 2010.

32. You N, Liu W, Wang T, et al: Swainsonine inhibits growth and potentiates the cytotoxic effect of paclitaxel in hepatocellular carcinoma in vitro and in vivo. Oncol Rep 28: 2091-2100, 2012.

33. Hu S, Sun W, Wei W, et al: Involvement of the prostaglandin $\mathrm{E}$ receptor EP2 in paeoniflorin-induced human hepatoma cell apoptosis. Anticancer Drugs 24: 140-149, 2013

34. Sun WY, Wang L, Liu H, Li X and Wei W: A standardized extract from Paeonia lactiflora and Astragalus membranaceus attenuates liver fibrosis induced by porcine serum in rats. Int J Mol Med 29: 491-498, 2012.

35. Thompson HJ, Strange R and Schedin PJ: Apoptosis in the genesis and prevention of cancer. Cancer Epidemiol Biomarkers Prev 1: 597-602, 1992.

36. Giannattasio A, Angeletti G, De Rosa M, et al: RNA expression bcl-w, a new related protein Bcl-2 family and caspase-3 in isolated sertoli cells from pre-pubertal rat testes. J Endocrinol Invest 25: C23-C25, 2002.

37. Adams JM and Cory S: The Bcl-2 protein family: arbiters of cell survival. Science 281: 1322-1326, 1998.

38. Cory S and Adams JM: The Bcl2 family: regulators of the cellular life-or-death switch. Nat Rev Cancer 2: 647-656, 2002.

39. Barille-Nion S, Bah N, Vequaud E and Juin P: Regulation of cancer cell survival by BCL2 family members upon prolonged mitotic arrest: opportunities for anticancer therapy. Anticancer Res 32: 4225-4233, 2012.

40. Zeng H, Kong X, Peng H, et al: Apoptosis and Bcl-2 family proteins, taken to chronic obstructive pulmonary disease. Eur Rev Med Pharmacol Sci 16: 711-727, 2012.

41. Kinoshita M, Eguchi Y and Hynynen K: Activation of Bak in ultrasound-induced, JNK- and p38-independent apoptosis and its inhibition by Bcl-2. Biochem Biophys Res Commun 353: 515-521, 2007.

42. Galluzzi L, Kepp O and Kroemer G: Caspase-3 and prostaglandins signal for tumor regrowth in cancer therapy. Oncogene 31: 2805-2808, 2012

43. Porter AG and Janicke RU: Emerging roles of caspase-3 in apoptosis. Cell Death Differ 6: 99-104, 1999.

44. Zhu S, Cohen MB, Bjorge JD, Mier JW and Cho DC: PI3K inhibition potentiates Bcl-2-dependent apoptosis in renal carcinoma cells. J Cell Mol Med 17: 377-385, 2013

45. Lin HI, Lee YJ, Chen BF, et al: Involvement of Bcl-2 family, cytochrome $\mathrm{c}$ and caspase 3 in induction of apoptosis by beauvericin in human non-small cell lung cancer cells. Cancer Lett 230: 248-259, 2005.

46. Tao YM, Liu Z and Liu HL: Dickkopf-1 (DKK1) promotes invasion and metastasis of hepatocellular carcinoma. Dig Liver Dis 45: 251-257, 2013

47. Korpi JT, Hagstrom J, Lehtonen N, et al: Expression of matrix metalloproteinases-2, -8, -13, -26 and tissue inhibitors of metalloproteinase-1 in human osteosarcoma. Surg Oncol 20: e18-e22, 2011. 